Bull. Mater. Sci., Vol. 14, No. 6, December 1991, pp. 1323-1329. (C) Printed in India.

\title{
A divergent beam diffraction method for study of single crystals
}

\author{
SATYAPAL SINGH and A M SRIRAMAMURTY \\ Defence Metallurgical Research Laboratory, Hyderabad 500 258, India \\ MS received 13 May 1991
}

\begin{abstract}
A divergent beam X-ray diffraction technique has been described for study of single crystals. This technique not only helps in observing defects in single crystals but also in determining the crystal orientation. Two methods have been proposed for observing images of sections of single-crystal components.
\end{abstract}

Keywords. Microfocus X-ray; single crystal; turbine blade; orientation determination; tomography.

\section{Introduction}

Single crystals of various materials are being produced for a variety of applications where either physical properties such as electronic, optical properties etc or their superior mechanical properties such as creep and tensile strength in case of superalloy single crystal are used. Often the quality of their performance depends on crystal orientation and freedom from defects. In the manufacture of components based on single crystal, one looks for a fast and easy technique for determining orientation and quality of the crystals.

This paper presents two techniques, one for the determination of orientation and the other for the formation of images of sections of crystals based on diffraction of a divergent beam of unfiltered X-rays. The recent advent of microfocus radiography systems is expected to make the above technique adoptable on a routine basis.

\section{Theory}

Consider a single crystal being illuminated with white radiation of X-rays emanating from a source $S$ as shown in figure 1. Since rays of all wavelengths in all directions are available the only condition to be satisfied for diffraction is that the incident and diffracted beams make the same angle with the diffracting crystal planes.

Now consider diffraction from crystal region along the plane normal $P_{1}$. Application of the above condition for diffraction shows that all rays diffracted by this crystal region converge to a point $I_{1}$ on the crystal plane passing through the source such that

$$
S P^{\prime}=P^{\prime} I_{1} \text {, i.e. } S I_{1}=2 S P^{\prime}
$$

where $P^{\prime}$ is the point of intersection of the plane normal considered with the plane passing through the source. This focussing phenomena is the same as the one described by Barrett and Massalski (1968). The intensity of the diffracted rays at $I_{1}$ depends on the amount and quality of the crystal matter along the plane normal exposed. Therefore $I_{1}$ may be considered as an image of the exposed crystal region 


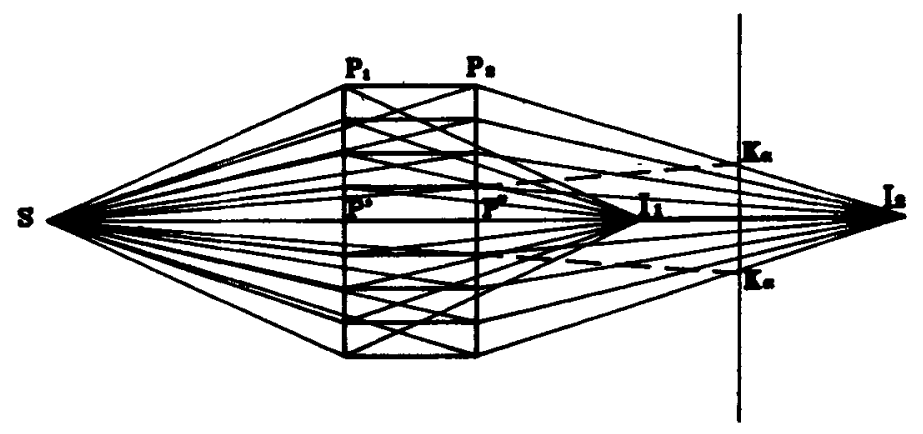

Figure 1. Formation of projected image of $2 x$ magnification due to focussing of diffracted $\mathrm{X}$-rays from a point source.

projected along the plane normal $P_{1}$. Consider now the crystal region along another plane normal $\boldsymbol{P}_{2}$. The diffracted beam due to this crystal region converges to a point $I_{2}$ on the crystal plane passing through source such that

$$
S P^{\prime \prime}=P^{\prime \prime} I_{2}, \quad S I_{2}=2 S P^{\prime \prime}
$$

The diffracted spot $I_{2}$ corresponds to the image of crystal region along plane normal $P_{2}$. For every plane normal between $\boldsymbol{P}_{1} \boldsymbol{P}_{2}$ there is a corresponding image formed between $I_{1} I_{2}$. Thus not only the intensity of the diffracted spots is related to the material along the corresponding plane normal but also there is a one-to-one correspondence between the location of the crystal normals and their diffracted spots. Therefore $I_{1} I_{2}$ is an image of the crystal as projected along the plane normal.

From equations (1) and (2) it may be seen that

$$
I_{1} I_{2}=2 P^{\prime} P^{\prime \prime},
$$

thus, the magnification of the image is $2 x$. Using the fact that the incident and diffracted rays and the plane normal are coplanar, it is easy to show that the magnification in the direction normal to the sketch in figure 1 is also $2 x$. Thus the image has an overall magnification of $2 x$. Further the image is formed at a distance twice that of the specimen from the source.

Thus diffracted rays from the whole of the crystal due to specific crystal plane focusses onto a plane parallel to the diffracting planes passing through the source $S$, which may be referred to as the image plane or focal plane. All crystal planes having non-zero structure factor will give rise to a corresponding focal plane image on the corresponding planes.

\section{Applications}

\subsection{Crystal orientation determination}

Let us consider a small crystal $C$ and examine formation of images by several planes due to X-ray beam from a source at $O$. The diffracted image is formed at $I$ such that normal to the diffracting planes $C N$ is the perpendicular bisector of the line $O I$ shown in figure 2. With this condition it is obvious that the diffracted images of all crystal planes will lie on a sphere with a radius equal to $O C$ with its 


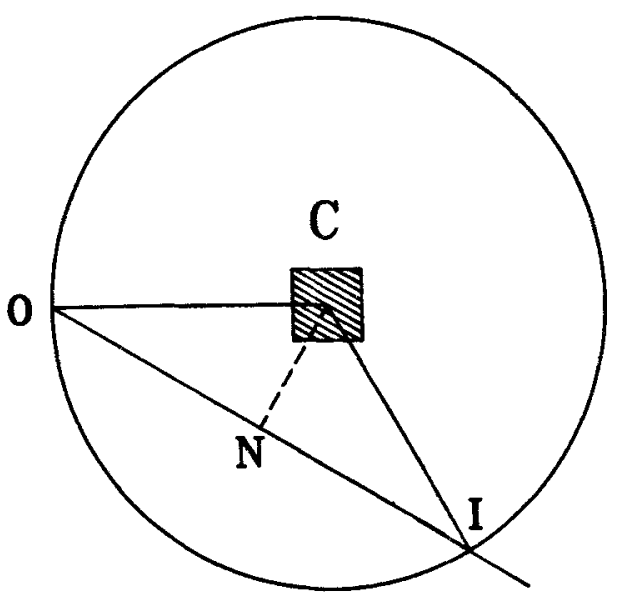

Figure 2. Position of image due to diffraction from planes at an arbitrary angle.

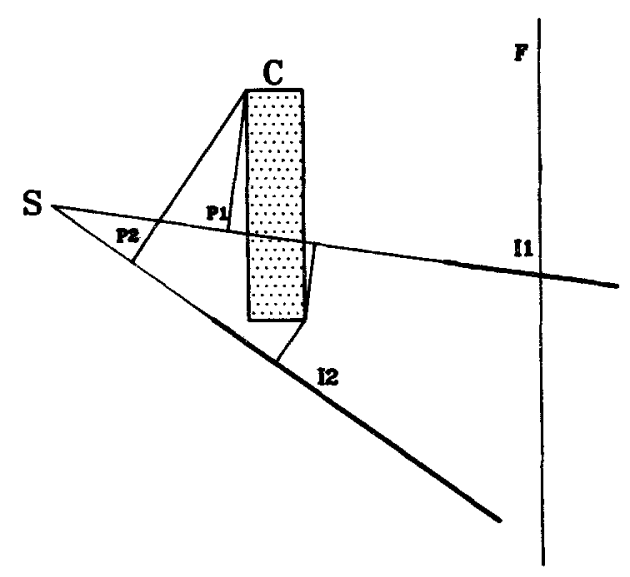

Figure 3. Condition that the focal plane should pass through the specimen for an image to cut the recording film.

centre at $C$. It would therefore be difficult to record the diffracted images of several planes on a planar photo film or imaging screen. On the other hand if we take a disc of single crystal it would be possible to record the traces of some diffracted planes on flat film placed parallel to the specimen surface and at a distance twice that of specimen from the source as shown in figure 3. By applying the fact that every crystal region forms a diffracted image on the focal plane at a distance twice as much as the distance between the source and specimen, it may be seen that only those diffracting planes whose focal planes pass through the specimen, have their image-cutting through the film and so form sharp lines on the film. Thus within the shadow of the specimen, on the film we get sharply focussed lines corresponding to each of the diffracting planes. It may be noted that these lines are intersections of the diffracting plane and the plane of the film.

Let us now consider the nature of traces recorded at the points of intersection of zone axis which happen to fall within the radiograph. More than one trace pass 


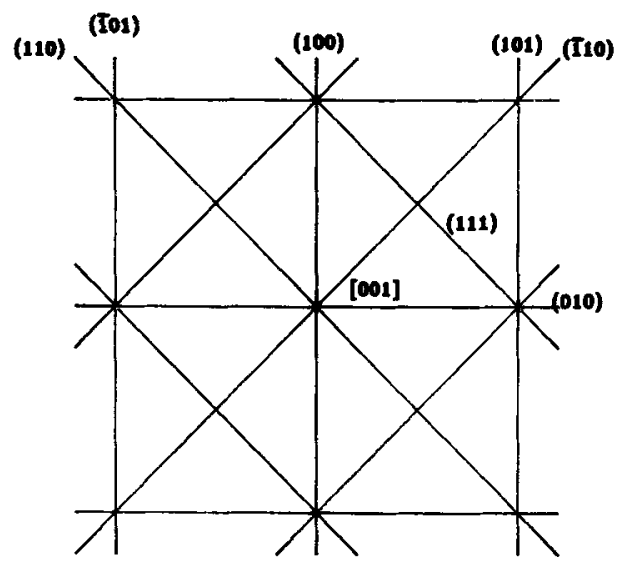

Figure 4. The pattern of traces of images formed on flat film when the source lies on [001] zone axis and normal to the film.

through such points and show the symmetry that exists about that axis especially when the zone axis is normal to the film. Figure 4 is the expected image that may be obtained from an fcc single-crystal disc with [001] zone axis normal to the film. Observe the four-fold symmetry at the centre. Such a symmetry about zone axes can help in identification of zone axis and determination of crystal orientation. The angular relation between the traces observed at other zone axis intersection which are away from the centre does not represent the true angular relations between the corresponding planes. They represent the true angle relationship only when the zone axis intersections is brought to the centre. Therefore it is necessary to bring the zone axis to the centre or close to. the centre by manipulation of the specimen for determining its symmetry. The recent advent of microfocus system with realtime imaging and remote specimen manipulation facility can speed up orientation determination by this technique. From the geometry shown in figure 3 it is clear that one should use the source-to-specimen distances comparable to the size of the specimen to image more than one zone axis at a time. Microfocus systems available today can allow use of such short source-to-specimen distances. That the image traces have enough intensity for being recorded is shown in figure 5 , the radiograph of a directionally-solidified columnar-grained turbine blade taken using a commercially available microfocus system. It may be observed that the central dark line is associated with two light lines on both sides, these are due to the loss of intensity caused by diffraction of $K_{\alpha}$ rays in that direction as shown in figure 1 . For determining the crystal orientation with respect to the external geometry of the specimen, the specimen mounted on manipulator may be manipulated while observing the diffraction lines to bring the zone axis to the centre. The angular displacement required to bring the zone axis to the centre gives the relation between the zone axis and the external geometry of the specimen.

\subsection{Orientation relationship/misorientation}

Every crystal in the specimen will give rise to a diffraction behaviour as discussed 


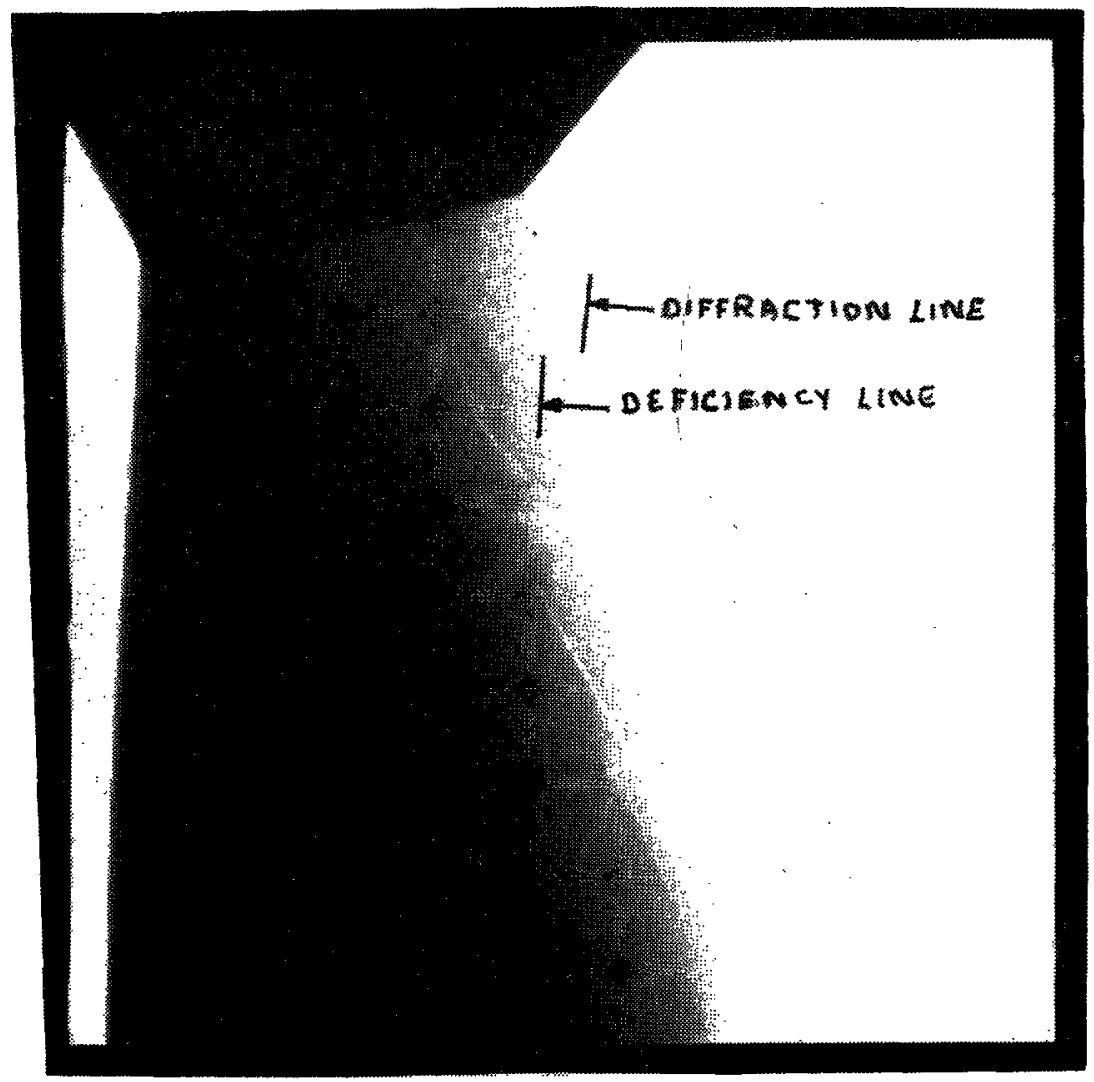

Figure 5. Radiograph of directionally-solidified columnar-grained turbine blade showing sharp lines due to the images of various planes and the associated deficient lines.

above. Thus if the single-crystal specimen considered happens to contain a second phase in the form of precipitate or a eutectic phase with a definite orientation relationship with the matrix, the second phase will form its own diffraction grid of traces of all reflecting planes. Since both the matrix and the precipitated form diffracted grids on to the film, it would be possible to determine the orientation relationship between two planes.

On the other hand if the specimen contains more than one grain or subgrains, each of the crystal will form its own diffraction grid from which one can determine the misorientations.

\subsection{Imaging sections of single crystals (tomography)}

As explained in $\S 2$ the diffracted rays from the crystal region along any given plane normal converges to a point on the focal plane. This phenomenon can be used to image sections of single-crystal components such as single-crystal turbine blades by two methods described below. 


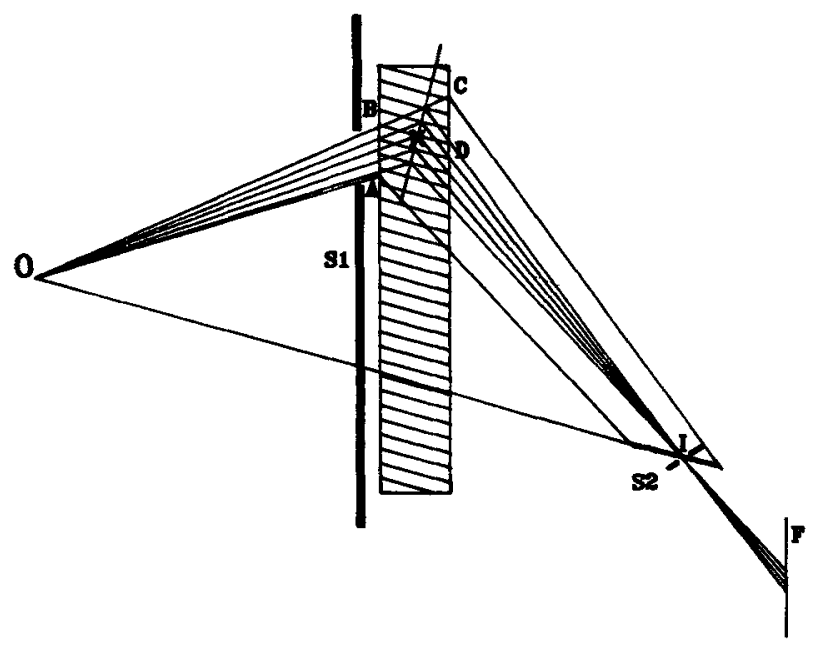

Figure 6. Arrangement for imaging of sections of single-crystal component.

\section{Method 1}

As explained earlier, the projected image of the crystal along the plane normal forms on the focal planes with a magnification $2 x$. Now consider a single-crystal component and an X-ray source $O$ positioned as shown in figure 6 . If we place a slit $S_{1}$ between the source and specimen allowing only a narrow fan of beam to illuminate the specimen, we get projected image $I_{1} I_{2}$ of only the illuminated region $A B C D$ of the specimen on the focal plane of the diffraction plane on which a film can be placed for recording. If the thickness of the beam fanning out of the slit is made small we get essentially a view of the section illuminated as projected along the diffracting plane normal.

The image thus obtained will show physical defects such as holes, inclusions and crystal defects such as misoriented regions, lattice distortion etc. The region where holes, inclusions or misoriented crystal are present does not diffract to the corresponding regions in the focal plane and consequently they appear as holes.

\section{Method 2}

Instead of placing a slit in the path of the incident beam, if one places a slit $S_{2}$ on the focal plane with its length perpendicular to the plane containing the diffracting plane normal and X-ray source and positioned such that it allows rays converging at one point such as $I N$ as shown in figure 6 , then the rays passing through the slit images the crystal region containing the plane normal $N$ and parallel to the length of the slit. Since no focussing of the beam is involved for imaging the magnification of the image produced by this method does not have any fixed magnification and depend on the distance of the film from the slit and source $O$. In this method the amount of crystal region imaged depends on the width of the opening in the slit $S_{2}$.

One application that one can think of for such a tomographic technique would 
be for imaging sections of advanced aircooled single-crystal gas turbine blades as an NDT tool for checking the position of the cooling channels.

\section{Acknowledgement}

The authors thank Dr P Rama Rao, Director, DMRL for his keen interest.

\section{Reference}

Barrett C S and Massalski T B 1968 Structure of metals (New Delhi: Eurasia Publishing House) III ed., p. 96 